\title{
Commentary
}

\section{Reflections of a Palliative Care Physician in the United Arab Emirates}

\author{
Neil Nijhawan, BSc (Hons), PhD, MBBS, MRCP* \\ Consultant in Palliative Medicine, Burjeel Medical City, $28^{\text {th }}$ Street, Mohamed Bin Zayed City, Abu Dhabi, UAE
}

\section{"Corresponding author}

Neil Nijhawan, BSc (Hons), PhD, MBBS, MRCP

Consultant in Palliative Medicine, Burjeel Medical City, 28 ${ }^{\text {th }}$ Street, Mohamed Bin Zayed City, Abu Dhabi, UAE; Tel. +97I 508663675 ;

E-mail: neil.nijhawan@burjeelmedicalcity.com

\section{Article information}

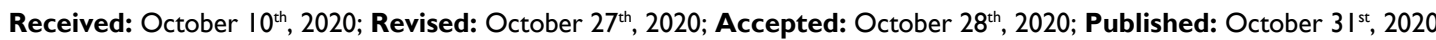

\section{Cite this article}

Nijhawan N. Reflections of a palliative care physician in the United Arab Emirates. Palliat Med Hosp Care Open J. 2020; 6(2): 19-21. doi: 10.17140/PMHCOJ-6-139

\section{INTRODUCTION |}

$\mathrm{T}$ The United Arab Emirates (UAE), along with the rest of the world, is facing a significant challenge in mitigating the spread of coronavirus disease 2019 (COVID-19) and the resulting pressure on frontline healthcare staff. At the time of writing, the worldwide total number of confirmed COVID-19 cases has exceeded 5 million with thankfully only a fraction of these $(\sim 37000)$ in the UAE. However, what is frightening many at this time is that so many of these patients did not survive. With access to 24-hour news and daily reports of worldwide mortality figures, not since the Second World War we have been reminded of our own mortality and the fragility of life in such a sustained manner.

As a palliative care doctor, my response to the question - "So what kind of doctor are you?" usually produces two responses. Half the time, the person I am speaking to will tell me that they couldn't possibly imagine doing my job and how am I not depressed dealing with all the death and dying. The other 50\% will respond with silence, raised eyebrows and wide-open eyes before rapidly trying to change the conversation. Since I recently moved to the UAE, the latter response rate is now probably up to $75 \%$. While I am not surprised by these frequent responses, it struck me that one of the best ways to make mortality less taboo and more accessible is to use my specialty, palliative medicine as a mean to promote more open and honest conversations.

Palliative care, as a medical specialty, is not restricted to patients with advanced cancer alone, rather, palliative care aims to ensure that patients with any potentially life-threatening illness achieve the best quality of life regardless of their diagnosis or prognosis. This is achieved by ensuring optimal control of symptoms (physical and psychological) and facilitating open, honest conversations about their hopes and fears. Strong support for their family and carers is also given. Palliative medicine's approach is pragmatic-planning for the worst but hoping for the best. Palliative care provision within the UAE remains limited to hospitals specialising in cancer treatment, namely: the American Hospital and Mediclinic in Dubai, Tawam Hospital in Al Ain, and most recently, at Burjeel Medical City in Abu Dhabi.

The COVID-19 pandemic has effectively presented us with a major-disaster type scenario whereby potentially many hundreds of patients may become critically unwell and die within a very short timeframe. High-risk patients (for example, the elderly and those with multiple comorbidities, including long-term immunosuppression, patients with an advanced cancer, diabetes, and advanced heart failure, lung disease' kidney disease) are palliative care's core patient demographic. My palliative care colleagues around the world have been extremely busy supporting teams caring for patients dying as a result of COVID-19 related complications. $^{1}$

The UAE has thankfully, not experienced the levels of COVID-related mortality seen in other parts of the world. However, as noted above, palliative care in the UAE is not as wellestablished as in other countries, and the current pandemic is highlighting the need to have more open conversations about our own mortality. There are a number of themes that are commonly encountered during palliative care consultations, and these are discussed in the following sections.

\section{“I'm Still Having Treatment, I Don't Need Palliative Care Yet”}

Although palliative care has traditionally been associated with the care of patients at the end-of-life, palliative care input is applicable and relevant at any point during serious illness. It is focused on the patient's needs rather than on their diagnosis or prognosis. So, if a patient has difficult to control symptoms, e.g., pain, breathlessness, nausea, anxiety, psychological distress to name but a few, palliative care can help with managing them. I may review a patient at multiple touch points throughout their cancer treatment journey 
or may only provide input once or twice on an as required basis. The rationale is that palliative care nurses and doctors focus on improving a patient's symptoms in order to produce an improved sense of well-being.

The skill of palliative care physicians and nurses is enabling patients to live as well for as long as possible, particularly towards the end of their life, by holistically addressing the needs of the person-physical, psychological and spiritual-as well of the needs of their family/carers.

Importantly, palliative care is not an alternative to standard medical treatment, it is given in conjunction to them. A landmark study in $2010^{2}$ demonstrated that patients receiving treatment for stage 4 lung cancer who also received palliative care input at the time of their diagnosis had better pain control, a reduced incidence of depression and improved quality of life. Moreover, these patients lived on average 3-months longer than patients who only received palliative care on an as-required basis. Palliative care therefore is clearly applicable at any point in a patient's illness not just towards the end-of-life.

\section{"Don't Give her Any Bad News"}

Family members will often insist that a patient should not receive bad news about their diagnosis, otherwise they may lose hope, become depressed and deteriorate sooner. It is completely understandable that families wish to protect their loved ones from harm (including the perceived psychological harm of a bad clinical diagnosis). However, this belief is not without risk.

Every patient is unique and therefore, the approach to their care must be individualised. Patients know best how they feel, and they will usually be aware that something is not quite right with their body. Patients have the right to know as much or as little as they wish to know about their condition. Often, patients will wait until they are alone with the medical team before asking for the truth about the extent of their illness.

Not facilitating the opportunity to openly discuss their illness and prognosis denies them the opportunity to have a detailed conversation about their wishes or preferences for further medical treatment. They may subsequently become increasingly anxious about their illness, and this anxiety may actually cause suffering if they feel that family members are not being honest with them.

Open conversations with a patient and their family can be emotional yet liberating experiences. After the tears have dried up, there is often a tangible sense of calm as the pressure to keep the illness (or it's extent) a secret is eliminated, and people are able to relax. Taking away or reducing uncertainty, although it may seem difficult to achieve, can be incredibly helpful to both the patient and their wider support network of family and close friends.

\section{Expectation Mismatch}

As a society, we have very high expectations of what modern medicine can deliver. Even in patients with a terminal illness, medicine has become adept at keeping us alive for longer. However, there often comes a point where many of us ask ourselves "just because we can do this, does that mean that we should, how is this actually helping the patient?'

It is natural for families to feel that they have tried everything possible to keep a loved one alive, but, there is an exceptionally fine line between subjecting a patient to increasingly invasive medical interventions and doing actual harm. In the UAE, we are comfortable with the concept that everything is in God's hands, and will regularly have to explain to families that God has enabled the medical team to get their loved one this far, but despite all that modern medicine has to offer, the body is still failing. In these situations, the language that we use is important and so we never talk about there being 'nothing else that we can do'. Instead, we focus on the aspects of care that we still have some degree of control over-ensuring that the patient is comfortable, symptoms are wellcontrolled, and they have the best quality of life for whatever time they have left-there is always something that we can do to make patients feel more comfortable.

When someone is deteriorating as a result of an illness, part of what makes it difficult to deal with is the loss of control, loss of physical independence, loss of the ability to make decisions. Loss of control over all aspects of your life is profoundly disorientating and unsettling. Relatives and carers can also experience this loss of control; palliative care attempts to ensure that they receive as much support as the patient.

\section{Opioid-Phobia}

Pain is rated as the most common symptom in patients with both cancer and non-cancer diagnoses. Palliative care physicians use opioids for the management of both severe pain and breathlessness, but fear of opioid drug addiction remains widespread. This fear is mainly due to many in the medical profession, the media and the general public not having a clear understanding of how to use of opioids for pain management. In the right hands, the use of opioids can be transformative for the patient.

Opioids are a group of medications that includes codeine, tramadol, morphine, oxycodone and fentanyl. When appropriately prescribed for severe pain, there are rarely adverse side effects and addiction is not an issue. We need to take a common-sense approach and realise that opioids, like all other classes of medications, have a role to play in improving the quality of life of patients and are safe and well-tolerated when correctly used. Pain is a universally awful experience and I always say to my patients that I will utilise all the medications that I have access to including morphine, to help minimise the pain and reduce their suffering.

\section{CONCLUSION}

As the COVID-19 pandemic continues to spread, we can take the opportunity to pause and reflect on the fragility of life. We are all mortal beings and we will all die at some point. While we may not have any control over our life span, we do have the ability to think about the kind of care that we would want for ourselves should we 
become seriously unwell with a life-threatening illness and discuss this with your doctors and your loved ones. Yes, this may be scary and intensely emotional, but open and honest conversations can relieve some of that fear and in stil feelings of peace and a sense of control. These are the sorts of conversations that palliative care is skilled at facilitating. While we may not always be able to cure all our patients, there is always something that we can do to make them feel better in themselves.

\section{AUTHOR CONTRIBUTIONS}

This article was conceived and written solely by the author.

\section{FUNDING SOURCES}

There was no funding associated with this article.

\section{REFERENCES |}

1. Radbruch L. de Lima L. de Joncheere C, Bhadelia A. The key role of palliative care in response to the COVID-19 tsunami of suffering. Lancet. 2020; 395: 10235: 1467-1469. doi: 10.1016/S01406736(20)30964-8

2. Temel JS, Greer JA, AlonaMuzikansky MA, Gallagher ER, Admane S; Jackson VA, et al. Early palliative care for patients with metastatic non-small cell lung cancer. N Engl J Med. 2010; 363: 733 742. doi: 10.1056/NEJMoa1000678 